\title{
Identification of fertility restorers, stable combiners and heterotic combinations across environments in pigeonpea [Cajanus cajanifolius (L.) Millsp.] in India
}

\author{
M. Byregowda*, H. Prashanthbabu and G.A. Maryreena
}

\begin{abstract}
Efficient and stable cytoplasmic genic male sterility system is a prerequisite for commercial exploitation of heterosis in any crop improvement program. The present investigation was undertaken to identify fertility restorers, stable combiners and best heterotic crosses in pigeonpea [Cajanus cajanifolius (L.) Millsp]. Totally, 75 crosses were developed by using three $\mathrm{A}_{4}$ cytoplasm-based male sterile lines viz., ICPA 2047, ICPA 2092 and ICPA 2089, and 25 diverse inbred lines. The pollen fertility assay identified 15 good restorers with $85-95 \%$ fertility status. The 45 , best restored combinations identified were assessed across four seasons (August and September, 2012 and 2013) for their stable heterosis and general and specific combining ability to identify parents possessing desirable genes. Variance for combining ability was significant $(p<.05)$ for lines, testers and line $\times$ tester effects. Among the three male sterile lines, ICPA 2047 was stable with a significant general combining ability for most of the traits followed by ICPA 2092, while the general combining ability effects among males was high across seasons for ICPL 20205 and ICPL 20177 ( $p<0.05$ ). The cross combination of ICPA $2089 \times$ ICPL 20205 had desirable genes for days to the first flowering, 50\% flowering and maturity. For yield related traits, cross combinations of ICPA $2047 \times$ ICPL 20128, ICPA $2092 \times$ ICPL 11376 and ICPA $2047 \times$ ICPL 20107 had significant specific combining ability indicating their usability in development of hybrids $(p<0.05)$. With respect to the heterotic ability of crosses, crosses involving parents ICPA 2089 as female and ICPL 88034 and ICPL 81-3 as males showed negative heterosis for flowering and maturity. For yield related traits, crosses with ICPA 2047 as the female parent and ICPL 20128, ICPL 20177 and ICPL 20096 as the male parents showed significant positive heterosis $(p<0.05)$. The identified parents with good combining ability and cross combination`s with significant heterosis could be utilized in producing high yielding hybrids in pigeonpea.
\end{abstract}

Keywords: CGMS, combining ability, fertility restoration, heterosis, pigeonpea

\section{Introduction}

Pigeonpea [Cajanus cajanifolius (L.) Millsp] is an important grain legume crop of rainfed agriculture in the semi-arid tropics. It is grown widely in the Indian subcontinent. The gross cultivated area under pigeonpea in India is 3.86 million ha with a total production of 2.65 million $\mathrm{mt}$ and a productivity of $686.5 \mathrm{~kg} \mathrm{ha}^{-1}$. In spite of the efforts made by the pigeonpea crop researchers for more than

All India Co-ordinated Research Project on Pigeonpea, University of Agricultural Sciences, Bangalore, India

* Corresponding Author: mbyregowda59@gmail.com 
five decades, the progress in the genetic improvement of yield in this crop is limited.

Hybrid pigeonpea is one of the potential methods to break this yield stagnation. The stable male-sterility system in conjunction with natural outcrossing will make the hybrid pigeonpea production easy and affordable. Effective utilization of a male-sterility system in hybrid breeding can be accomplished firstly by identification of stable restorers for the concerned male sterile lines over different environments. Secondly, it is also essential to narrow down male sterile lines, maintainer and restorer lines having high general combining ability for yield and its attributing traits and hybrid with desirable specific combining ability. Thirdly, is to identify a stable hybrids and ideal environment to realize the potential yield of pigeonpea. The present study was thus carried out to identify fertility restorers, stable combiners and best heterotic crosses in pigeonpea.

\section{Materials and Methods}

The experimental material for the restoration study comprised of six $A_{4}$ cytoplasm-based CMS lines of pigeonpea viz., ICPA 2047, ICPA 2092, ICPA 2089 (developed at the International Crops Research Institute for Semi-Arid Tropics, Hyderabad, India), BRG $1 A$, BRG 3A, Hy3 A (developed at the University of Agricultural Sciences, Bangalore, India) and 61 pollen parents. A total of 75 cross combinations were produced in line $\times$ tester mating design with three female (ICPA 2047, ICPA 2092 and ICPA 2089) and 25 male parents. Similarly, 108 combinations were produced between three female (BRG 1A, BRG 3A and $\mathrm{Hy} 3 \mathrm{CA}$ ) and 36 male parents. All cross combinations were evaluated along with parents and two checks (ICPH 2671 and ICP 8863) in a randomized complete block design (RCBD) with two replicates in 2012. For testing the pollen fertility in the hybrids, a $2 \%$ acetocarmine stain was used and pollens were examined under light microscope to differentiate the fertile and sterile pollens. The mean per cent pollen fertility of hybrids was calculated (Equation 1).

Pollen fertility $(\%)=\frac{\text { No. of fertile }(\text { round and stained) pollens }}{\text { Total number of pollen grains studied }} \times$ x

Based on the fertility restoration in the hybrids, 45 hybrid combinations involving three male sterile lines (ICPA 2047, ICPA 2092 and ICPA 2089) and 15 restorers were selected for studies leading to combining ability and heterosis. The 45 hybrids along with the check (ICPH 2671) were planted in a RCBD with two replicates during four seasons in August and September of 2012 and 2013. Observations were recorded on nine quantitative traits on randomly selected five competitive plants on each of the hybrids for estimating combining ability 
and heterosis. The overall status of hybrids across of the traits was determined using the method proposed by Arunachalum et al. (1984).

\section{Results and Discussion}

The frequency of restorers for different $A_{4}$ cytoplasm-based male sterile lines is shown in Tables 1 and 2. In total, 56 pollen parents were found to be restoring fertility of hybrids (more than $80 \%$ ). Among the different cross combinations studied, 14 genotypes (56\%) were found to be the restorer for ICPA 2047, 12 genotypes (48\%) for ICPA 2092, eight genotypes (32\%) for ICPA 2089, six genotypes ( $17 \%$ ) for BRG $3 A$ and eight genotypes each with $22 \%$ frequency restored fertility in the hybrid combinations with BRG 1 Aand Hy3CA. Basha et al. (2008), Saxena (2009) and Makelo et al. (2013) reported such variations when tested under varying environmental conditions.

Table 2 indicates that 12 genotypes were the common restorers for ICPA 2047 and ICPA 2092, eight each for ICPA 2047 and ICPA 2089, ICPA 2092 and ICPA 2089, ICPA 2047, ICPA 2092 and ICPA 2089, three for BRG 1A and BRG 3A; four each for BRG $1 A$ and $\mathrm{Hy}_{3} \mathrm{Ca}$; $B R G 3 A$ and $\mathrm{Hy}_{3} \mathrm{CA}$, and two for $B R G 1 A$, BRG $3 A$ and $H y 3 C A$. The genotype ICPL 20093 was the common restorer for all the six male sterile lines.

Table 1. Frequency of restorers of six $\mathrm{A}_{4}$ cytoplasm based male sterile lines in pigeonpea

\begin{tabular}{lccc}
\hline CMS Lines & $\begin{array}{c}\text { Number of } \\
\text { pollen } \\
\text { parents }\end{array}$ & $\begin{array}{c}\text { No. of restorers } \\
\text { with }>80 \% \\
\text { pollen fertility }\end{array}$ & $\begin{array}{c}\text { Frequency of } \\
\text { restorers (\%) }\end{array}$ \\
\hline ICPA 2047 & 25 & 14 & 56 \\
ICPA 2092 & 25 & 12 & 48 \\
ICPA 2089 & 25 & 8 & 32 \\
BRG 1 A & 36 & 8 & 22 \\
BRG 3A & 36 & 6 & 17 \\
Hy 3C A & 36 & 8 & 22 \\
\hline
\end{tabular}

The 45 hybrid combinations were analyzed for combining ability of their parents and heterosis across four seasons. The analysis of variance (Table 3 ) revealed significant differences between gca effects of CMS lines for all the traits except for pods per plant. In case of pollen parents, effects of gca differed significantly for days to maturity, plant height, secondary branches and 100 seed weight. The male sterile line ICPA 2089 was the best general combiner with negative and significant gca effects for days to 50\% flowering and maturity, while ICPA 2047 was a desirable combiner for yield and its related traits (Table 4). 
Byregowda et al.

Table 2. Common restorers for six $\mathrm{A}_{4}$ cytoplasm based male sterile lines in pigeonpea

\begin{tabular}{|c|c|c|}
\hline CMS Lines & $\begin{array}{l}\text { No. of } \\
\text { restorers }\end{array}$ & Restorers \\
\hline ICPA 2047 \& ICPA 2092 & 12 & $\begin{array}{l}\text { ICPL 161, ICPL 11376, ICPL 20093, ICPL 20096, } \\
\text { ICPL 20098, ICPL 20108, ICPL 20128, CPL 20166, } \\
\text { ICPL 20205, ICPL 81-3, ICPL 87119 \& ICPL } 88039\end{array}$ \\
\hline ICPA 2047 \& ICPA 2089 & 8 & $\begin{array}{l}\text { ICPL 11376, ICPL 20093, ICPL 20096, ICPL 20098, } \\
\text { ICPL 20166, ICPL 20205, ICPL 81-3 \& ICPL } 87119\end{array}$ \\
\hline ICPA 2092 \& ICPA 2089 & 8 & $\begin{array}{l}\text { ICPL 11376, ICPL 20093, ICPL 20096, ICPL 20098, } \\
\text { ICPL 20166, ICPL 20205, ICPL 81-3 \& ICPL } 87119\end{array}$ \\
\hline $\begin{array}{l}\text { ICPA 2047, ICPA } 2092 \\
\& \text { ICPA } 2089\end{array}$ & 8 & $\begin{array}{l}\text { ICPL 11376, ICPL 20093, ICPL 20096, ICPL 20098, } \\
\text { ICPL 20166, ICPL 20205, ICPL 87119 \& ICPL 81-3 }\end{array}$ \\
\hline$B R G 1 A \& B R G 3 A$ & 3 & TS 3R, ICPL 20093 \& BRGL 13-171 \\
\hline BRG $1 A \&$ Hy $3 C$ A & 4 & GCB 126, ICPL 81-3, ICPL 20093 \& BRGL 13-171 \\
\hline BRG $3 \mathrm{~A} \& \mathrm{Hy} 3 \mathrm{C} \mathrm{A}$ & 4 & $\begin{array}{l}\text { ICPL 20093, ICPL 20116, BRGL 13-167 \& BRGL 13- } \\
171\end{array}$ \\
\hline $\begin{array}{l}\text { BRG } 1 \text { A, BRG } 3 \\
A \& H y 3 C A\end{array}$ & 2 & ICPL 20093 \& BRGL 13-171 \\
\hline
\end{tabular}

Amongst the pollen parents, ICPL 20128 and ICPL 161 have negative and significant gca effects on days to 50\% flowering and maturity, while ICPL 20128 and ICPL 20177 showed significant and positive gca effects on yield and its related traits ( $p<0.05)$. Dalvi et al. (2008), Yadav et al. (2008) and Sameerkumar et al. (2009) observed similar results with parents having high gca effects, which has ultimately resulted in hybrids with superior sca effects.

The estimates of specific combining ability effects of 45 hybrids are summarized in Table 5. The hybrid ICPA $2047 \times$ ICPL 20098 and ICPA $2092 \times$ ICPL 88034 were identified as the best specific combiners in desirable direction for days to $50 \%$ flowering and maturity. The hybrid combinations, ICPA $2092 \times$ ICPL 20128 and ICPA $2089 \times$ ICPL 20205 for plant height; ICPA $2047 \times$ ICPL 20098 and ICPA $2092 \times$ ICPL 20108 for primary branches, ICPA $2089 \times$ ICPL 161 and ICPA $2089 \times$ ICPL 81-3 for secondary branches, ICPA $2047 \times$ ICPL 20128 and ICPA 2047 $\times$ ICPL 161 for number of pods per plant, ICPA $2047 \times$ ICPL 20128 and ICPA $2047 \times$ ICPL 20093 for number of seeds per pods, ICPA $2089 \times$ ICPL 88034 and ICPA $2089 \times$ ICPL 88039 for 100 seed weight were the best specific combinations in desirable combinations.

For seed yield per plant, the hybrids ICPA $2092 \times$ ICPL 11376 and ICPL ICPA $2047 \times$ ICPL 20128 were the best specific combinations. Dalvi (2008), Yadav et al. (2008) and Sameerkumar et al. (2009) also reported similar results that could lead to hybrids with superior sca effects. 
Table 3. Analysis of variance for combining ability effects across environments in pigeonpea

\begin{tabular}{|c|c|c|c|c|c|c|c|c|c|c|}
\hline $\begin{array}{l}\text { Sources of } \\
\text { variation }\end{array}$ & $\mathrm{df}$ & $\begin{array}{r}\text { Days to } \\
50 \% \\
\text { flowering } \\
\end{array}$ & $\begin{array}{l}\text { Days to } \\
\text { maturity }\end{array}$ & $\begin{array}{r}\text { Plant } \\
\text { height } \\
(\mathrm{cm})\end{array}$ & $\begin{array}{r}\text { Primary } \\
\text { branches/ } \\
\text { plant } \\
\end{array}$ & $\begin{array}{r}\text { Secondary } \\
\text { branches/ } \\
\text { Plant }\end{array}$ & Pods/plant & $\begin{array}{r}\text { Seeds/ } \\
\text { pod }\end{array}$ & $\begin{array}{r}100 \text { seed } \\
\text { weight } \\
(\mathrm{g}) \\
\end{array}$ & $\begin{array}{r}\text { Seed } \\
\text { yield/plant } \\
(\mathrm{g}) \\
\end{array}$ \\
\hline Replication & 1 & $2939.0 * *$ & 4.0 & 4.0 & $24.3^{* *}$ & $17.9^{*}$ & 357.8 & 0.1 & 2.0 & 103.7 \\
\hline Hybrids & 44 & $4296.5^{* *}$ & $1726.5^{* *}$ & $1726.5^{* *}$ & $10.5^{* *}$ & $21.5 * *$ & $18939 \cdot 9^{* *}$ & $0.2^{* *}$ & $4 \cdot 3^{* *}$ & $365.1 * *$ \\
\hline Line & 2 & $65632.6 * *$ & $26372.0 * *$ & $26372.0 * *$ & $93.8^{* *}$ & $247.9 * *$ & 46708.3 & $0.7^{* *}$ & $16.1^{* *}$ & $2167.1 * *$ \\
\hline Tester & 14 & 1607.7 & $1392.4 * *$ & $1392.4 * *$ & $9 \cdot 3$ & $20.3^{* *}$ & 24129.1 & 0.2 & $6.7 * *$ & 312.8 \\
\hline Line * Tester & 28 & $1259.7^{* *}$ & $133.1 * *$ & $133.1 * *$ & $5.2 * *$ & $5 \cdot 9^{* *}$ & $14361.9 * *$ & $0.1 * *$ & $2.3^{* *}$ & $262.6 * *$ \\
\hline Env * Hybrids & 132 & $2099 \cdot 9 * *$ & $266.5^{* *}$ & $266.5^{* *}$ & $5.6 * *$ & $9 \cdot 9^{* *}$ & $4179.6 * *$ & 0.04 & $2.1 * *$ & $217.8 * *$ \\
\hline Env * Line & 6 & $21415.2 * *$ & $759 \cdot 4^{* *}$ & $759 \cdot 4^{* *}$ & $34.8 * *$ & $31.3^{* *}$ & $10909.5 *$ & 0.07 & 1.7 & 355.4 \\
\hline Env * Tester & 42 & 1190.8 & 242.9 & 242.9 & 4.1 & 10.1 & 2337.8 & 0.04 & 2.3 & $155 \cdot 9$ \\
\hline Error & 176 & 158.5 & 10.2 & 10.2 & 2.3 & 2.9 & 625.7 & 0.05 & 0.9 & 42.9 \\
\hline $\operatorname{Var}(G C A)$ & & 464.8 & 192.7 & 112.8 & 0.7 & 1.8 & 484.5 & 0.01 & 0.2 & 16.7 \\
\hline $\operatorname{Var}(\mathrm{SCA})$ & & 138.6 & 15.4 & 13.7 & 0.3 & 0.4 & 1728.2 & 0.01 & 0.2 & 28.1 \\
\hline $\begin{array}{l}\operatorname{Var}(\mathrm{GCA}) / \text { Var } \\
(\mathrm{SCA})\end{array}$ & & 3.35 & 12.49 & 8.21 & 2.19 & 4.55 & 0.28 & 0.77 & 0.8 & 0.59 \\
\hline
\end{tabular}

icant at $\mathrm{p}=0.05, * *$ Significant at $\mathrm{p}=0.01$ 
Table 4. Estimates of gca effects of parents across four environments for nine traits in pigeonpea

\begin{tabular}{lllr}
\hline Parental lines & Characters & Cenotypes & gca effects \\
\hline CMS Lines (Seed & Days to 50 \% flowering & ICPA 2089 & -13.03 \\
parents) & Days to maturity & ICPA 2089 & -16.99 \\
& Plant height (cm) & ICPA 2047 & 18.48 \\
& Primary branches/plant & ICPA 2047 & 0.63 \\
& Secondary branches/plant & ICPA 2047 & 0.74 \\
& Pods/plant & ICPA 2047 & 21.03 \\
& Seeds/Pod & ICPA 2047 & 0.07 \\
& 100 Seed Weight (g) & ICPA 2047 & 0.29 \\
& Seed yield/ plant (g) & ICPA 2047 & 4.46 \\
& Days to 50\% flowering & ICPL 20128 & -5.62 \\
& Days to maturity & ICPL 161 & -11.46 \\
& Plant height (cm) & ICPL 20128 & 12.96 \\
& Primary branches/ plant & ICPL 20177 & 0.71 \\
& Secondary branches/plant & ICPL 20108 & 1.34 \\
& Pods/plant & ICPL 20177 & 68.82 \\
& Seeds/Pod & ICPL 20098 & 0.12 \\
& 10o Seed Weight (g) & ICPL 20093 & 0.80 \\
& Seed yield/ plant (g) & ICPL 20177 & 5.02 \\
\hline
\end{tabular}

Table 5. Best three hybrids based on sca effects and their per se performance for nine traits in pigeonpea

\begin{tabular}{|c|c|c|c|c|}
\hline Characters & Hybrids & sca & Mean & $\begin{array}{c}\text { Standard } \\
\text { heterosis over } \\
\text { ICPH } 2671\end{array}$ \\
\hline Days to $50 \%$ & ICPA $2047 \times$ ICPL 20098 & -6.09 & 100.50 & 15.85 \\
\hline \multirow[t]{2}{*}{ flowering } & ICPA $2047 \times$ ICPL 20128 & -5.42 & 90.23 & 4.30 \\
\hline & ICPL $2092 \times$ ICPL 88034 & -5.02 & 89.75 & 3.46 \\
\hline \multirow[t]{3}{*}{ Days to maturity } & ICPA $2092 \times$ ICPL 88034 & -6.13 & 130.25 & -3.25 \\
\hline & ICPA $2047 \times$ ICPL 20098 & -5.93 & 152.00 & 12.91 \\
\hline & ICPA $2089 \times$ ICPL 20205 & -4.80 & 128.25 & -4.74 \\
\hline \multirow[t]{3}{*}{ Plant height $(\mathrm{cm})$} & ICPA $2092 \times$ ICPL 20108 & 19.38 & 177.85 & $17 \cdot 30$ \\
\hline & ICPA $2089 \times$ ICPL 20205 & 19.13 & 144.92 & -4.42 \\
\hline & ICPA $2047 \times$ ICPA 88034 & 17.11 & 178.20 & 17.53 \\
\hline Primary branches/ & ICPA $2092 \times$ ICPL 20108 & 0.88 & 9.58 & 33.27 \\
\hline \multirow[t]{2}{*}{ Plant } & ICPA $2047 \times$ ICPL 20098 & 0.95 & 10.27 & 42.82 \\
\hline & ICPA $2047 \times$ ICPL 20205 & 1.26 & 9.78 & 36.03 \\
\hline Secondary branches/ & ICPA $2092 \times$ ICPL 20128 & 1.18 & 5.67 & 116.73 \\
\hline \multirow[t]{2}{*}{ Plant } & ICPA $2089 \times$ ICPL 81-3 & 1.51 & 1.03 & -60.80 \\
\hline & ICPA $2089 \times$ ICPL 161 & 1.83 & 1.69 & -35.28 \\
\hline \multirow[t]{3}{*}{ Pods/Plant } & ICPA $2089 \times$ ICPL 20107 & 59.07 & 225.09 & 19.09 \\
\hline & ICPA $2047 \times$ ICPL 161 & 64.29 & 244.85 & 29.55 \\
\hline & ICPA $2047 \times$ ICPL 20128 & 66.05 & 285.40 & 51.00 \\
\hline \multirow[t]{3}{*}{ Seeds/Pod } & ICPA $2047 \times$ ICPL 20128 & 0.14 & 3.80 & 6.37 \\
\hline & ICPA $2089 \times$ ICPL 20093 & 0.14 & 3.80 & 6.37 \\
\hline & ICPA $2047 \times$ ICPL 20107 & 0.20 & 3.93 & 9.94 \\
\hline
\end{tabular}




\begin{tabular}{llllc}
\hline 100 Seed Weight (g) & ICPA 2089 × ICPL 88039 & 0.73 & 10.66 & -4.76 \\
& ICPA 2092 × ICPL 20098 & 0.76 & 10.95 & 3.23 \\
& ICPA 2089 × ICPL 88034 & 0.79 & 10.62 & -5.17 \\
Seed yield/plant (g) & ICPA 2089 × ICPL 20166 & 6.19 & 37.34 & 11.81 \\
& ICPA 2047 × ICPL 20128 & 11.67 & 54.72 & 63.85 \\
& ICPA 2092 × ICPL 11376 & 14.73 & 50.73 & 51.92 \\
\hline
\end{tabular}

Amongst the 45 hybrids, ICPA $\times$ ICPL 20128 and ICPA $2092 \times$ ICPL 11376 were the most heterotic over the standard check ICPH 2761 for seed yield per plant. Sekhar et al. (2004) and Wanjari et al. (2007) reported substantial heterosis for grain yield and other economic characters over the best checks in pigeonpea.

Twenty out of 45 hybrids recorded an overall high sca status over environments (Table 6 ). From these 20 hybrids, which showed higher overall sca status, four were of $\mathrm{H} \times \mathrm{H}$, five with $\mathrm{H} \times \mathrm{L}$, four with $\mathrm{L} \times \mathrm{H}$ and remaining seven with $L \times L$ combinations. In addition to determining the overall gca status of parents and sca status of hybrids, it is important to determine overall heterotic status of hybrids across the traits (Table 7). It is evident from the table 7 that 23 out of 45 hybrids had high $(\mathrm{H})$ heterotic status and the remaining hybrids had low (L) overall heterotic status across the traits.

Table 6. Overall specific combining ability status of hybrids for nine traits in pigeonpea

\begin{tabular}{lcccccc}
\hline \multirow{2}{*}{$\begin{array}{l}\text { Lines/ Testers with } \\
\text { overall gca }\end{array}$} & \multicolumn{2}{c}{ ICPA 2047 (H) } & \multicolumn{2}{c}{ ICPA 2092 (L) } & \multicolumn{2}{c}{ ICPA 2089 (L) } \\
\cline { 2 - 7 } & $\begin{array}{c}\text { Total } \\
\text { score }\end{array}$ & $\begin{array}{c}\text { Over all } \\
\text { heterotic } \\
\text { status }\end{array}$ & $\begin{array}{c}\text { Total } \\
\text { score }\end{array}$ & $\begin{array}{c}\text { Over all } \\
\text { heterotic } \\
\text { status }\end{array}$ & $\begin{array}{c}\text { Total } \\
\text { score }\end{array}$ & $\begin{array}{c}\text { Over all } \\
\text { heterotic } \\
\text { status }\end{array}$ \\
\hline ICPL 20108 (L) & 151 & $\mathrm{~L}$ & 371 & $\mathrm{H}$ & 245 & $\mathrm{~L}$ \\
ICPL 20166 (L) & 201 & $\mathrm{~L}$ & 239 & $\mathrm{~L}$ & 356 & $\mathrm{H}$ \\
ICPL 11376 (L) & 207 & $\mathrm{~L}$ & 376 & $\mathrm{H}$ & 139 & $\mathrm{~L}$ \\
ICPL 161 (L) & 328 & $\mathrm{H}$ & 174 & $\mathrm{~L}$ & 222 & $\mathrm{~L}$ \\
ICPL 87119 (H) & 281 & $\mathrm{H}$ & 285 & $\mathrm{H}$ & 198 & $\mathrm{~L}$ \\
ICPL 20205 (H) & 180 & $\mathrm{~L}$ & 249 & $\mathrm{~L}$ & 305 & $\mathrm{H}$ \\
ICPL 20098 (L) & 340 & $\mathrm{H}$ & 184 & $\mathrm{~L}$ & 258 & $\mathrm{H}$ \\
ICPL 20096 (L) & 258 & $\mathrm{H}$ & 211 & $\mathrm{~L}$ & 272 & $\mathrm{H}$ \\
ICPL 20093 (H) & 243 & $\mathrm{~L}$ & 226 & $\mathrm{~L}$ & 316 & $\mathrm{H}$ \\
ICPL 81-3 (L) & 211 & $\mathrm{~L}$ & 299 & $\mathrm{H}$ & 239 & $\mathrm{~L}$ \\
ICPL 20128 (H) & 399 & $\mathrm{H}$ & 198 & $\mathrm{~L}$ & 164 & $\mathrm{~L}$ \\
ICPL 88039 (L) & 214 & $\mathrm{~L}$ & 349 & $\mathrm{H}$ & 198 & $\mathrm{~L}$ \\
ICPL 20107 (H) & 209 & $\mathrm{~L}$ & 188 & $\mathrm{~L}$ & 363 & $\mathrm{H}$ \\
ICPL 88034 (H) & 265 & $\mathrm{H}$ & 268 & $\mathrm{H}$ & 231 & $\mathrm{~L}$ \\
ICPL 20177 (H) & 256 & $\mathrm{H}$ & 183 & $\mathrm{~L}$ & 336 & $\mathrm{H}$ \\
\hline
\end{tabular}

Final norm: 253, (H) - High overall general combiner, (L) - Low overall general combiner, $\mathrm{H}$ - High overall specific combination, L - Low overall specific combination 
Byregowda et al.

Table 7. Overall heterotic status of hybrids for nine traits in pigeonpea

\begin{tabular}{lcccccc}
\hline \multirow{2}{*}{$\begin{array}{l}\text { Lines/ Testers } \\
\text { with overall gca }\end{array}$} & \multicolumn{2}{c}{ ICPA 2047 (H) } & \multicolumn{2}{c}{ ICPA 2092 (L) } & \multicolumn{2}{c}{ ICPA 2089 (L) } \\
\cline { 2 - 7 } & $\begin{array}{c}\text { Total } \\
\text { score }\end{array}$ & $\begin{array}{c}\text { Over all } \\
\text { heterotic status }\end{array}$ & $\begin{array}{c}\text { Total } \\
\text { score }\end{array}$ & $\begin{array}{c}\text { Over all } \\
\text { heterotic status }\end{array}$ & $\begin{array}{c}\text { Total } \\
\text { score }\end{array}$ & $\begin{array}{c}\text { Over all } \\
\text { heterotic status }\end{array}$ \\
\hline ICPL 20108 (L) & 164 & $\mathrm{~L}$ & 336 & $\mathrm{H}$ & 323 & $\mathrm{H}$ \\
ICPL 20166 (L) & 155 & $\mathrm{~L}$ & 270 & $\mathrm{H}$ & 335 & $\mathrm{H}$ \\
ICPL 11376 (L) & 147 & $\mathrm{~L}$ & 313 & $\mathrm{H}$ & 166 & $\mathrm{~L}$ \\
ICPL 161 (L) & 272 & $\mathrm{H}$ & 260 & $\mathrm{H}$ & 300 & $\mathrm{H}$ \\
ICPL 87119 (H) & 231 & $\mathrm{~L}$ & 323 & $\mathrm{H}$ & 313 & $\mathrm{H}$ \\
ICPL 20205 (H) & 142 & $\mathrm{~L}$ & 241 & $\mathrm{~L}$ & 292 & $\mathrm{H}$ \\
ICPL 20098 (L) & 182 & $\mathrm{~L}$ & 163 & $\mathrm{~L}$ & 229 & $\mathrm{~L}$ \\
ICPL 20096 (L) & 187 & $\mathrm{~L}$ & 250 & $\mathrm{~L}$ & 301 & $\mathrm{H}$ \\
ICPL 20093 (H) & 119 & $\mathrm{~L}$ & 235 & $\mathrm{~L}$ & 227 & $\mathrm{~L}$ \\
ICPL 81-3 (L) & 115 & $\mathrm{~L}$ & 202 & $\mathrm{~L}$ & 220 & $\mathrm{~L}$ \\
ICPL 20128 (H) & 385 & $\mathrm{H}$ & 277 & $\mathrm{H}$ & 356 & $\mathrm{H}$ \\
ICPL 88039 (L) & 178 & $\mathrm{~L}$ & 304 & $\mathrm{H}$ & 260 & $\mathrm{H}$ \\
ICPL 20107 (H) & 199 & $\mathrm{~L}$ & 237 & $\mathrm{~L}$ & 344 & $\mathrm{H}$ \\
ICPL 88034 (H) & 311 & $\mathrm{H}$ & 331 & $\mathrm{H}$ & 363 & $\mathrm{H}$ \\
ICPL 20177 (H) & 241 & $\mathrm{~L}$ & 268 & $\mathrm{H}$ & 318 & $\mathrm{H}$ \\
\hline
\end{tabular}

Final norm: 253; (H) - Overall high general combiner, (L) - Overall low general Combiner. $\mathrm{H}$ - Overall high specific combination, L - Overall low specific combination

\section{References}

Arunachalum, V., Bandopadhayay, A., Nigam, S.N. and Gibbons, R.W. (1984): Heterosis in relation to genetic divergence specific combining ability in groundnut Arachis hypogaea. Euphytica 33: 33-39

Basha, S.H., Byregowda M. and Girish G. (2008): Evaluation of new cytoplasmic male sterile lines in pigeonpea. Environ. Ecol. 26 (3A): 1353- 1355.

Dalvi, V.A., Saxena, K.B. and Madrap, I.A. (2008): Fertility restoration in cytoplasmic nuclear male-sterile lines derived from 3 wild relatives of pigeonpea. J. Heredity. 99(6): 671-673.

Makelo, M.N., Melis, R. and Girhiri, M. (2013): Stability of cytoplasmic male-genic sterility in pigeonpea [Cajanus cajan (L.) Millsp.] under different environmental conditions in Kenya. Int. J. Agric. Policy and Res. 1: 11-018.

Sameerkumar, C.V., Sreelaxkmi, C.H., Shivani, D. and Suresh, M. (2009): Study of heterosis for yield and its component traits in pigeonpea. J. Res. ANGRAU. 37 (3\&4): 86-91.

Saxena, K.B. (2009): A hybrid pea for the drylands. Appropriate Tech. 36(2): 38-39.

Sekhar, M.R., Singh, R.B., Mehra, S.P. and Govil, J.N. (2004): Combining ability and heterosis in early maturing pigeonpea [Cajanus cajan (L.) Milisp.] hybrids. Indian J. Genet. 64 (3): $212-216$.

Wanjari, K.B., Bhangle, S.A. and Sable, N.H. (2007): Evaluation of heterosis in CMS based hybrids in pigeonpea. J. Food Leg. 20(1): 107-108.

Yadav, A.S., Tank, C.J., Acharya, S. and Patel, J.B. (2008): Combining ability analysis involving Indo-African genotypes of pigeonpea. J. Food Leg. 21(2): 95-98. 